\title{
Tensile Stress and Plastic Deformation in Aluminum Induced by Aqueous
}

\section{Corrosion}

Ömer Özgür Çapraz ${ }^{\mathrm{a},}$, Shinsuke Ide ${ }^{\mathrm{a}}$, Pranav Shrotriya ${ }^{\mathrm{b}}$, and Kurt R. Hebert ${ }^{\mathrm{a},{ }^{*}}$

aDepartment of Chemical and Biological Engineering, Iowa State University, Ames, IA 50011, USA

${ }^{\mathrm{b}}$ Department of Mechanical Engineering, Iowa State University, Ames, IA 50011, USA

Keywords: stress measurement; corrosion; aluminum; morphological stability analysis;

hydrogen embrittlement; stress corrosion cracking; vacancies

* Corresponding author

E-mail address: krhebert@iastate.edu (K. R. Hebert) 


\begin{abstract}
Measurement of near-surface stress generated by metallic corrosion can reveal defects and material property changes relevant to structural degradation by stress corrosion cracking. In this study, stress and topography evolution were characterized during alkaline corrosion of high-purity aluminum. In situ stress measurements revealed tensile increases of the force per width (stress integrated over depth) that scale directly with the sample yield stress. Spectral analysis of the uniform dimpled surface pattern produced by corrosion showed that transient changes of its characteristic wavelength track closely with the force per width. Together, the stress and topography measurements imply that corrosion creates a plastically deformed metal layer. Tensile stress is attributed to the lattice contraction associated with metal vacancies introduced during dissolution. In agreement with this hypothesis, a vacancy diffusion model successfully predicted the time dependence and magnitude of the force response, as well as the observed scaling relation between plastic layer thickness and pattern wavelength. The driving force for vacancy formation is thought to arise from high hydrogen and low aluminum chemical potentials near the corroding surface, the latter imposed by the high dissolution potential relative to the equilibrium potential of aluminum.
\end{abstract}




\section{Introduction}

Corrosion processes in combination with external or residual stress can induce embrittlement of structural metals, leading to premature and unexpected failure by stress corrosion cracking (SCC). While SCC has been studied intensively for several decades because of its wide economic significance, critical chemical interactions responsible for corrosion-enhanced degradation have not been fully clarified. In some systems such as high-strength 7xxx series Al alloys, corrosion is accompanied by absorption of hydrogen produced by cathodic water reduction, and SCC may be closely related to hydrogen embrittlement (HE) [1]. SCC and HE of Al alloys has been explained in terms of mechanisms such as hydrogen-enhanced local plasticity (HELP), hydrogen-enhanced decohesion (HEDE) or brittle hydride fracture [2, 3]. Aside from hydrogen defects, several authors suggest that metal vacancies produced during corrosion may influence SCC and HE of various alloys. Vacancies are thought to promote degradation by facilitating dislocation emission or climb at crack tips [4-6], decohesion [7], or void formation [8-10]. Formation of vacancies has been explained by direct injection into the metal by dissolution [4-6, 11], tensile strain or cold work [9, 10]. Also, strong binding of vacancies to absorbed hydrogen can result in dramatically enhanced concentrations of both vacancies and hydrogen near corroding interfaces, potentially assisting failure by HELP and HEDE mechanisms [12-14]. On the other hand, it is thought that vacancy injection during dissolution is kinetically challenged by the appreciable energy barrier to form lattice vacancies, so that high electrochemical potentials at the dissolving surface may be required [15]. Recent advances in atomistic simulations have led to the 
development of quantitatively predictive models for SCC and HE that include relevant length scales ranging from crack dimensions down to those of chemical interactions between atoms at crack tips [16-19]. While these models can simulate the detailed effects of defects on embrittlement, there remains a critical need to understand the metalenvironment interactions during corrosion that control near-surface concentrations of defects participating in SCC.

In this study, we characterize formation of near-surface defects using in situ measurements of stress changes during corrosion. Hydrogen absorption and vacancy injection respectively expand and contract the metal lattice close to the surface, generating biaxial near-surface stress that in turn induces changes of sample curvature. High-resolution curvature measurements permit sensitive detection of near-surface hydrogen and vacancies during corrosion [20,21]. Chu and co-workers, using ex situ stress measurements, already found evidence that corrosion-induced tensile stress correlates with SCC susceptibility $[22,23]$. The present work focuses on defect generation during uniform alkaline corrosion of large-grain high-purity Al. Such samples avoid complications arising from localized corrosion processes at grain boundaries and second-phase particles. Alkaline corrosion of Al is accompanied by rapid hydrogen absorption $[14,24]$, as well as formation of large numbers of subsurface nanoscale voids, the latter which may be attributable to condensation of near-surface vacancies produced during dissolution [25-27].

The present experiments employ a new technique, phase-shifting curvature interferometry, with significantly higher curvature resolution than traditional cantilever defection methods [28, 29]. The enhanced curvature resolution permits stress 
measurements in relatively thick sheet and foil samples, in contrast to thin metal films typically used for deflection techniques. Thicker samples enable study of material property effects in experiments with extended corrosion exposures. The present measurements revealed large tensile stress increases that scale directly with the sample yield strength. Spectral analysis of the dimpled topography produced by dissolution demonstrated that the characteristic wavelength of this uniform surface pattern scales with corrosion-induced stress, apparently the first indication of corrosion-induced plastic deformation. Model calculations show that both the thickness of the plastically deformed layer and the pattern length scale are determined by the diffusion penetration of vacancies injected by dissolution. The electrochemical driving force for vacancy diffusion-induced tensile stress and plasticity is explained in terms of the high hydrogen chemical potential and low aluminum chemical potential imposed respectively by the metal dissolution and hydrogen absorption processes. 


\section{Experimental Methods}

Aluminum samples were fabricated from either $1 \mathrm{~mm}$ thick sheet of $99.998 \%$ purity (Alfa Aesar), or $25 \mu \mathrm{m}$ thick foil of $99.999 \%$ purity (Alfa Aesar). Electron backscatter diffraction revealed a typical grain size of 200 to $400 \mu \mathrm{m}$ in the sheet material. Some of the sheet samples were cold-worked to $0.65 \mathrm{~mm}$ thickness using a hand mill. Foils were annealed at $550^{\circ} \mathrm{C}$ for $52 \mathrm{hr}$, increasing their grain size to the order of $100 \mu \mathrm{m}$. Sample yield stress was estimated from the measured Vickers hardness (Wilson Tukon Hardness Tester) [30]. Usually the aluminum surface was not treated before dissolution. In one experiment, the aluminum sheet was electropolished for $5 \mathrm{~min}$ at $30 \mathrm{~V}$ in a solution of $20 \%$ perchloric acid in ethanol. Alkaline corrosion of the Al samples was at open circuit in aqueous sodium hydroxide solutions. Topographic changes resulting from dissolution were characterized by field-emission SEM (FEI Quanta 250) and atomic force microscopy (AFM, Veeco Metrology Dimension 3100).

Detailed descriptions of the phase shifting curvature interferometry system and procedures used in stress measurements are provided elsewhere [29]. For stress measurements, both sheet and foil samples were cut into rectangular shape $(3.5 \times 2.5 \mathrm{~cm})$. Only one side of the sample was exposed to the corrosive solution, while curvature changes were determined by interfering light beams reflected on the opposite side. Thus, curvature measurements are not affected by corrosion-induced surface roughening. To produce reflective surfaces for interferometry, gold films were transferred to one side of sheet samples using a template-stripping technique. Foils were bonded with epoxy to silicon wafers, which reflected the light beam and also functioned as elastically stiff 
substrates. Curvature and force per unit width changes are related by the thin-film Stoney approximation, $\Delta F_{w}=\left[E_{s} h_{s}^{2} / 6\left(1-v_{s}\right)\right] \Delta \kappa$, where $\Delta F_{w}$ and $\Delta \kappa$ are the force per width and curvature change; and $h_{s}, E_{s}$ and $v_{s}$ are the thickness, elastic modulus and Poisson's ratio of the $\mathrm{Al}$ sheet or $\mathrm{Si}$ wafer. $\Delta F_{w}$ represents the biaxial in-plane stress $\sigma_{x x}$ integrated through the sample thickness, relative to that before corrosion, with the $x$-direction parallel to the $\mathrm{Al}$ surface. 


\section{Results and Discussion}

\subsection{Stress measurements}

All force measurements revealed significant tensile stress increases during alkaline corrosion. Figure 1 compares stress evolution of $\mathrm{Al}$ sheet samples in $0.1,0.5$ and 1.0 $\mathrm{M} \mathrm{NaOH}$. Force per width in the figure represents the biaxial in-plane stress $\left(\sigma_{x x}\right.$, with $x$-direction parallel to surface) integrated through the near-surface stress-affected layer, relative to that before corrosion. In the $1.0 \mathrm{M}$ solution, the force increases initially in the compressive direction to a minimum value of to $-3 \mathrm{~N} / \mathrm{m}$ at $0.8 \mathrm{~min}$, after which tensile force begins to accumulate. Force change in the 0.1 and $0.5 \mathrm{M}$ solutions is tensile from the beginning of dissolution. In each solution, tensile force increases for 7 to $17 \mathrm{~min}$ until a plateau is reached. The plateau force increases significantly with concentration, but the rate of force increase before the plateau is similar in all three solutions. The rates of both metal dissolution and accompanying hydrogen absorption increase significantly with $\mathrm{pH}$ over the range in Figure 1 [24], suggesting that the stress response is controlled directly by the corrosion process.

Since Al has a moderate yield stress on the order of $100 \mathrm{MPa}$, tensile stress generated during corrosion could induce near-surface plastic deformation of aluminum metal. Figure 2 shows the force per width transient in $1 \mathrm{M} \mathrm{NaOH}$ for three samples with close to $99.999 \%$ purity but different yield stress: annealed $25 \mu \mathrm{m}$ foil, $1 \mathrm{~mm}$ as-received sheet and the same sheet after cold rolling to $650 \mu \mathrm{m}$ thickness. The respective yield strengths of the annealed foil, as-received sheet and rolled sheet were estimated as 20,90 
and $120 \mathrm{MPa}$ using Vickers hardness measurements. The force transient of the annealed foil but not the rolled sheet exhibits a compressive minimum of $-3 \mathrm{~N} / \mathrm{m}$ at $1 \mathrm{~min}$, similar to that of the as-received sheet. Both the average rate of tensile force increase and the plateau force increase significantly with yield stress. Indeed, the inset shows that the plateau force per width measured at 15 min scales with the yield stress, suggesting that tensile strain introduced by corrosion may be accommodated by near-surface plastic deformation.

\subsection{Correlation of topography and stress evolution}

In this section we examine the relationship between force evolution and the development of the dimpled or scalloped surface pattern produced by alkaline etching. Examples of scanning electron microscopy (SEM) images after dissolution times of 5 and 20 min are shown in Figure $3(a, b)$. Uniformly distributed characteristic dimpled depressions are found which increase in width over time up to $20 \mathrm{~min}$. AFM revealed that the dimple features are concave spherical sections with radius as large as $500 \mathrm{~nm}$. The radius of curvature of the convex ridges between dimples is much smaller, roughly 20 $30 \mathrm{~nm}$. These ridge and dimple dimensions are typical of patterns produced by etching or chemical polishing of aluminum [31, 32]. Spectral analysis of the SEM images was carried out to determine the characteristic length scales of the dissolution-induced morphology. Figure 3 (c) displays the Fast Fourier Transform (FFT) of the 20 min image in Figure $3(\mathrm{~b})$. The abscissa and ordinate are the spatial wavenumbers $1 / \lambda_{x}$ and $1 / \lambda_{y}$ of the Fourier modes of the image, where $\lambda_{x}$ and $\lambda_{y}$ are wavelengths in the horizontal and 
vertical directions. The color scale in the transform image is determined by the Power Spectral Density (PSD). The relatively bright spot in the central part of the image contains the modes contributing appreciably to the SEM image contrast. The inclination of the spot is caused by the preferred orientation of dimples along rolling marks on the sheet. Figure 3 (d) represents a line section along the long dimension of the spot in Figure 3 (c). The wavelength of the maximum PSD amplitude along the section corresponds to the characteristic width of dimple features.

Characteristic dimple widths after dissolution of sheet samples are compiled in Figure 4 and compared to the force measurements. The main panel shows the wavelengths on the left axis plotted against dissolution time, while the force transient in 1 $\mathrm{M} \mathrm{NaOH}$ is plotted for comparison using the right axis. The wavelength increases from $100 \mathrm{~nm}$ at $1 \mathrm{~min}$ to a stable range of 900 to $1.0 \mu \mathrm{m}$ reached after $15 \mathrm{~min}$. The transient increase of wavelength closely parallels that of force per width. For the lower $\mathrm{NaOH}$ concentrations, the wavelengths measured at 15 min lie below those for $1 \mathrm{M} \mathrm{NaOH}$. However, it is also appropriate to plot these data points at the times at the beginning of the plateaus, based on the constant wavelengths along the force plateau for $1 \mathrm{M} \mathrm{NaOH}$. Figure 4 shows that when this is done, all the wavelength data lie close to a common trajectory. Thus, all experiments initially follow a universal increasing trend of force or wavelength with time until reaching a steady state level, which is larger at higher $\mathrm{NaOH}$ concentration. The inset of Figure 4 shows explicitly the linear dependence of force on wavelength for as-received sheet samples.

The scaling of the pattern wavelength with the force change suggests that the dimpled pattern is produced by deformation. The uniformity of this pattern across the 
metal surface shows that the surface deformation is distributed uniformly. Therefore, the results of stress and topography measurements establish that (i) corrosion leads to uniform surface deformation, and (ii) this deformation coincides with the development of internal stresses that scale with the sample yield stress (Figure 2). These findings suggests that corrosion produces a uniform surface-adjacent plastically deformed layer. The measured force is dominated by this plastic layer, within which the stress is approximately equal to the yield stress. Similar near-surface plastic layers have been observed to form in Si wafers, as a result of tensile stress induced by dopant diffusion [33]. At steady state, the plastic layer thickness would be given by the slope of the inset graph in Figure 2, $\delta_{P S} \cong F_{W S} / \sigma_{Y}$, where $F_{W S}$ is the steady state force and $\sigma$ is yield stress. The similarity of the regression slope of $120 \mathrm{MPa}$ in Figure 4 and the yield stress of $90 \mathrm{MPa}$ suggests that the dimple width and plastic layer thickness are comparable to one another.

According to the plastically deformed surface layer model, the force transients would be interpreted as the increase of the plastic layer thickness with dissolution time. Normalized force defined as the ratio of force per width to yield stress would then estimate the layer thickness at a given time. Figure 5 plots the normalized force during dissolution of the various sample types in $1 \mathrm{M} \mathrm{NaOH}$. The duplicate experiments demonstrate the good reproducibility of the stress measurements. Normalized force transients for sheet and rolled sheet seem to collapse onto the same time dependence, which would support the interpretation of the force transient as plastic layer growth initiating near the onset of dissolution. However, both the steady-state plastic layer thickness and the time to steady state for foil samples are seen to be significantly smaller 
compared to sheet. These differences are addressed below with reference to model calculations. Also, Figure 5 shows that electropolishing the sheet prior to corrosion exposure does not significantly affect the force trajectory. Electropolishing Al sheet removes damaged surface layers produced by rolling and surface-segregated impurities such as $\mathrm{Pb}$, while increasing enrichments of bulk noble impurities like $\mathrm{Cu}[34,35]$. Insensitivity of force curves to these aspects of surface condition is consistent with tensile stress generation by Al dissolution itself.

\subsection{Stress generation mechanisms}

In this section, we discuss the origin of the stress changes accompanying corrosion. Compressive stress during the first minute of dissolution in some experiments can be explained by volume expansion caused by absorption of hydrogen interstitials [21], which occurs at high rates during alkaline corrosion $[14,24,36]$. Tensile stress increases at later times are caused by lattice contraction resulting from corrosion. Contraction contributed by diffusion of atoms from grain boundaries, while potentially significant in thin films [37], should be of minor importance in the present experiments because of the large grain size of the Al samples. Volume contractions would also be induced if dissolution creates metal vacancies replacing metal atoms $[14,36,38]$, as the vacancy volume is $35 \%$ smaller than that of $\mathrm{Al}$ atoms [39]. A near-surface vacancy concentration of $7 \times 10^{-3}$ would be sufficient to produce elastic stress equivalent to the yield stress. As mentioned above, vacancy injection is consistent with evidence from positron annihilation spectroscopy (PAS), transmission electron microscopy (TEM) and 
SEM that shows extensive formation of near-surface voids during alkaline dissolution [25-27]. More than $10^{8} \mathrm{~cm}^{-2}$ voids of width 10 to $20 \mathrm{~nm}$ were detected within a depth of about $80 \mathrm{~nm}$ after dissolution of $\mathrm{Al}$ foils in $1 \mathrm{M} \mathrm{NaOH}$ [27]. PAS measurements at dissolution times up to 20 min revealed voids, suggesting continuous generation of vacancies during the present experiments.

Prior theoretical work indicates that attractive hydrogen-vacancy interactions can lead to vacancy formation in $\mathrm{Al}$ at high hydrogen concentration. Density functional theory (DFT) calculations indicate that hydrogen occupancy of as many as 12 octahedral interstitial sites neighboring the vacancy is energetically favorable $[8,12,13,40]$. Thermodynamic models based on DFT-derived energetics indicate that large concentrations of vacancy-hydrogen defects should be present at equilibrium with the hydrogen concentrations on the order of 0.1 at. \% typically found during alkaline corrosion $[12-14,24,36]$. Proximity to the metal surface may further enhance vacancy formation [41]. Fukai and co-workers found experimental support for H-induced vacancy formation in a number of metals at high temperatures and $\mathrm{H}_{2}$ pressures [42]. We showed that hydrogen trapping at vacancies induced by hydrogen absorption can help explain hydride formation during alkaline dissolution [43]. On the contrary, other evidence suggests that hydrogen interactions alone would not account for the observed tensile stress increases. Recent stress measurements during cathodic polarization in acid solutions found near-surface concentrations of hydrogen on the order of 0.1 at. \% similar to that during alkaline corrosion, but detected only compressive stress due to interstitial hydrogen. In addition, the thermodynamic models show that Vac-H defects at equilibrium with high hydrogen concentration consist of multiple $\mathrm{H}$ atoms at interstitial 
sites around the vacancy [12]. Recent DFT modeling of H-vacancy interactions in Ni demonstrates that the formation volume of such defects is likely to be positive [44]. Creation of these equilibrium Vac-H defect would then result in volume expansion and compressive stress. Accordingly, it is unlikely that tensile stress generation as observed in the present experiments can be explained only in terms of elevated near-surface hydrogen concentration.

To reconcile the observed tensile stress with the high concentrations of both vacancies and hydrogen near the corroding surface, it is necessary to additionally consider effects of metal dissolution on subsurface defects. The primary anodic reaction during corrosion is the oxidation of $\mathrm{Al}$ atoms to form $\mathrm{Al}^{+3}$ ions incorporated into the $\mathrm{Al}(\mathrm{OH})_{3}$ surface film; at the same time, aluminate ions dissolve from this film into the saturated alkaline solution [45]. Also, analytical evidence from Secondary Ion Mass Spectroscopy and Atom Probe Tomography (APT) indicate continuing formation of aluminum hydride $\left(\mathrm{AlH}_{3}\right)$ during alkaline dissolution $[46,47]$. The presence of hydride at the $\mathrm{Al} / \mathrm{Al}(\mathrm{OH})_{3}$ interface is consistent with findings that the $\mathrm{pH}$-dependence of the dissolution potential tracks closely with that of the Nernst potential of hydride oxidation $[48,49]$. APT images of the interfacial region indicated that hydride is dispersed at the nanoscale, as opposed to being segregated in isolated domains, thus implying that the same electrochemical potential applies at both $\mathrm{Al}$ and $\mathrm{AlH}_{3}$ sites [50]. Expressing this potential in terms of both the $\mathrm{Al}$ and $\mathrm{AlH}_{3}$ oxidation reactions yields the constraint,

$$
\mu_{A l}-\mu_{A l}^{0}=3 F\left(E_{A l}^{0}-E_{\mathrm{AlH}_{3}}^{0}\right)+3 F \eta
$$


where $\mu_{\mathrm{Al}}$ is the $\mathrm{Al}$ atom chemical potential, $E_{\mathrm{Al}}^{0}$ and $E_{\mathrm{AlH}_{3}}^{0}$ are the standard potentials of $\mathrm{Al}$ and $\mathrm{AlH}_{3}$ oxidation to $\mathrm{Al}(\mathrm{OH})_{3}$, and $\eta$ is the kinetic overpotential for $\mathrm{Al}$ oxidation. Equation 1 assumes that both $\mathrm{Al}(\mathrm{OH})_{3}$ and $\mathrm{AlH}_{3}$ are at their standard chemical potentials, as implied by the close proximity of the dissolution potential to the Nernst potential of hydride oxidation. The standard potential difference in Equation 1 is $-0.6 \mathrm{~V}$ in Equation 1 $[48,51]$, while a study of alkaline dissolution kinetics on fractured Al surfaces suggests that $\eta$ is limited to values of about $0.1 \mathrm{~V}$ [52]. Therefore $\mu_{A l}-\mu_{A l}^{0}$ in Equation 1 is about $-1.5 \mathrm{eV}$. This $\mu_{\mathrm{Al}}$ deficiency is more than twice the formation energy of bulk vacancies in Al [13], and therefore by itself would produce large equilibrium vacancy concentrations. In the dissolution experiments, vacancy formation is additionally promoted by the elevated hydrogen concentration.

In summary, the discussion in this section argues that in the alkaline corrosion process there are two important driving forces for vacancy formation: elevated $\mu_{\mathrm{H}}$ and deficient $\mu_{\mathrm{Al}}$. Our recent stress measurements on cathodically polarized Al suggest that high $\mu_{H}$ alone would produce compressive stress associated with hydrogen interstitials and possibly vacancies bound to multiple $\mathrm{H}$ atoms [21]. On the other hand, the deficient $\mu_{\mathrm{Al}}$ imposed by the high potential of $\mathrm{Al}$ dissolution would tend to promote high concentrations of vacancies with a reduced hydrogen binding, consistent with volume contraction and tensile stress as revealed by the present measurements.

\subsection{Prediction of diffusion penetration of vacancies}


To test the vacancy injection hypothesis, the penetration depth of vacancy diffusion was estimated and compared to the plastic layer thickness from stress measurements. Injected vacancies would diffuse into the metal, generating a defect layer which grows in thickness over time until a steady state is reached at which the rates of vacancy formation and removal by dissolution are balanced. As a first approximation, we do not include stress effects on diffusion, since stress gradients should be damped within the plastic layer. Thus, the concentration of vacancies evolves according to the diffusion equation, $\partial C_{V} / \partial t=D_{V} \partial^{2} C_{V} / \partial z^{2}$, where $z$ is the depth coordinate on a stationary reference frame and $D_{V}$ is the vacancy diffusivity. The vacancy concentration at the dissolving metal surface is taken as a constant value $C_{0}$ as implied by Equation 1, and the concentration is set to zero both initially and at large depths. Transforming to dimensionless variables $\tau=v_{d}^{2} t / D_{V}, x=v_{d}\left(z-v_{d} t\right) / D_{V}$, and $c_{V}=C_{V} / C_{0}$, where $v_{d}$ is the approximately constant dissolution velocity, the diffusion equation becomes $\partial c_{V} / \partial \tau-\partial c_{V} / \partial x=\partial^{2} c_{V} / \partial x^{2}$, and the boundary condition at $x=0$ is $c_{V}=1$. Model calculations show that the vacancy concentration profile evolves to a steady-state exponential shape in which diffusion and dissolution are balanced, $c_{V}(\tau \rightarrow \infty)=e^{-x}$ (Figure 6). The defect layer thickness $x_{D}(\tau)$, defined by $c_{V}\left(x_{D}\right) / c_{V}(x=0)=0.2$, increases to a steady state value of 1.5 reached at a time of $\tau_{S} \cong 4$. The steady state defect layer corresponds to the force plateaus in Figures 1-2 and 5.

The vacancy diffusion model was used to predict the main features of the experimental force transients. First we consider the different force evolution of sheet and foil samples in Figure 5. The dimensional defect layer thickness at steady state is 
inversely proportional to the dissolution rate, $\delta_{D S} \cong 1.5 D_{V} / v_{d}$. Noting that the dissolution rate of foil in $1 \mathrm{M} \mathrm{NaOH}(190 \mathrm{~nm} / \mathrm{min})$ [24] is larger than that of sheet $(131 \mathrm{~nm} / \mathrm{min})$, the steady state defect layer thickness of foil should be smaller by a factor of 131/190 or 0.69. This ratio is nearly the same as the ratio of normalized forces in Figure 5. The dissolution rate also influences the time to steady state, $t_{S} \cong 4 D_{V} / v_{d}^{2}$, which should be smaller for foil than sheet by a factor of $(131 / 190)^{2}$ or 0.48 . Indeed, according to Figure 5, the experimental times to steady state of foils are about one-half those of sheet. The model also rationalizes the dependence of the force transients in Figure 1 on $\mathrm{NaOH}$ concentration. The vacancy diffusivity was estimated from both $t_{s}\left(D_{V} \cong v_{d}^{2} t_{s} / 4\right)$ and the steady-state force $F_{W S}\left(D_{V} \cong v_{d} F_{W S} / 1.5 \sigma_{Y}\right)$. Table 1 shows that these two methods result in closely similar diffusivity values at the three $\mathrm{NaOH}$ concentrations, which are also on the same order of magnitude as the vacancy diffusivity extrapolated from high temperature measurements, $4 \times 10^{-16} \mathrm{~m}^{2} / \mathrm{s}$ [53-55]. The agreement between force transients and model calculations is compelling evidence that vacancy diffusion accounts for the observed tensile stress evolution. The increase of diffusivity with $\mathrm{pH}$ in Table 1 is likely caused by factors neglected in the present simplified model, such as effects of stress or vacancy complexation on diffusion. The last column of Table 1 shows that the steady-state pattern wavelength and plastic layer thickness are comparable at all three $\mathrm{NaOH}$ concentrations tested.

\subsection{Morphological stability analysis}


In this section, we examine whether morphological stability analysis based on vacancy diffusion-induced plastic flow predicts the observed scaling of pattern wavelength with plastic layer thickness. Since plastic deformation accommodates strain induced by vacancy diffusion, we relate the metal lattice velocity at the interface to the vacancy diffusion flux, $\mathbf{v} \square \mathbf{n}_{\mathbf{I}}=\frac{1}{3} f_{V} \Omega \mathbf{J}_{\mathbf{V}} \square \mathbf{n}_{\mathbf{I}}$. Here $\mathbf{n}_{\mathbf{I}}$ is the interface normal vector, $f_{V}$ is the fractional lattice contraction of vacancies, and $\Omega$ is the molar volume of aluminum atoms. The interface velocity is augmented by the lattice velocity, $\mathbf{v}_{\mathbf{I}} \square \mathbf{n}_{\mathbf{I}}=v_{d}+\frac{1}{3} f_{V} \Omega \mathbf{J}_{\mathbf{V}} \square \mathbf{n}_{\mathbf{I}}$, where $v_{d}$ is the dissolution velocity. $v_{d}$ is given by $v_{d}=\Omega R_{A l} /\left(1+f_{V} C_{0}\right)$, where $R_{A l}$ is the metal dissolution rate and $C_{0}$ is the vacancy concentration at the interface. At the scale of the dimpled pattern, $R_{A l}$ should be spatially uniform, since the solution diffusion layer thickness is much larger than the dimple height [56]. Given a constant $C$, the diffusion flux and thus the interface velocity are enhanced on valleys and reduced at peaks, thereby causing interface perturbations to grow. Thus, plastic flow driven by vacancy diffusion destabilizes the flat interface. Restricted vacancy diffusion beneath ridges in the dimple pattern further explains observations of preferential nucleation of voids near ridges [27].

Pattern formation during dissolution requires the existence of a stabilizing process along with destabilizing plastic flow. Given the high interface curvature at ridges, we suggest that surface energy considerations would tend to stabilize the interface by promoting plastic flow toward the bulk metal. Surface forces would be significant when the capillary pressure in the metal is comparable to the yield stress. This is indeed the case, as the ridge radius of curvature of order $10 \mathrm{~nm}$ and the surface free energy of order 
$1 \mathrm{~N} / \mathrm{m}$ indicate a capillary pressure of $100 \mathrm{MPa}$, the same order of magnitude as the yield stress of the Al samples. We capture this in a simple way by appending a curvaturedependent term to the interface velocity,

$$
\mathbf{v}_{\mathbf{I}} \square \mathbf{n}_{\mathbf{I}}=v_{d}(1+a \kappa)+\frac{1}{3} f_{V} \Omega \mathbf{J}_{\mathbf{V}} \square \mathbf{n}_{\mathbf{I}}
$$

The parameter $a$ is a length parameter expressing the sensitivity of the interface velocity to curvature. The local surface curvature is defined by the two-dimensional Laplacian operator as $\kappa=\nabla_{1}^{2} z_{I}$, where $z I(x, y, t)$ is the local interface position. Note that $v_{d} a \kappa$ is interpreted as an enhancement of plastic flow, despite the grouping of this factor with the dissolution velocity.

We again assume as a first approximation that vacancy diffusion is driven primarily by concentration as opposed to stress gradients. Thus, the vacancy diffusion flux in equation 2 is calculated from the vacancy concentration distribution as determined by the diffusion equation, $\partial C_{V} / \partial t=D_{V} \nabla^{2} C_{V}$, where $C_{V}$ is the vacancy concentration. The contribution of the small lattice velocity to diffusion is neglected. The boundary conditions for $C_{V}$ are $C_{V}=C_{0}$ at $z=Z_{I}$ and $C_{V} \rightarrow 0$ as $Z \rightarrow \infty$.

The linear stability approach is used to model the response of the interface profile $Z_{I}(x, y, t)$ to small perturbations. Accordingly, $C_{V}$ and $z_{I}$ are perturbed slightly from the basic state condition of steady-state dissolution of a flat metal surface:

$C_{V}(x, y, z, t)=\bar{C}_{V}(z)+C_{V}^{\prime}(x, y, z, t)$, where the overbar denotes the basic state and the prime indicates the perturbation. To solve for the basic state concentration, the reference 
frame is changed to that of the dissolving interface, $\zeta=z-\bar{Z}_{I}(t)$. The time dependence of $\bar{Z}_{I}$ is given by $\bar{Z}_{I}=v_{I} t$, where the basic state interface velocity is $v_{I}=v_{d} /\left(1-f_{V} C_{0} / 3\right)$

. The basic state vacancy concentration profile is the same as in the one-dimensional diffusion model, $\bar{C}_{V}(\zeta)=C_{0} \exp \left(-v_{I} \zeta / D_{V}\right)$. The time dependence of the perturbed variables is determined by linearizing the model about the basic state. In terms of dimensionless variables, the linearized diffusion equation is $\partial C^{\prime} / \partial \tau-\partial C^{\prime} / \partial s=\nabla^{2} C^{\prime}$ where $C=C_{V} / C_{0}, s=v_{d} \zeta / D_{V}$ and $\tau=v_{d}^{2} t / D_{V}$. The interface boundary condition is derived by writing $C$ at the perturbed surface in terms of the value at the reference plane $s$ $=0$,

$$
\left.C\right|_{s_{I}^{\prime}}=\left.\bar{C}\right|_{0}+\left.C^{\prime}\right|_{0}+\left.\frac{d \bar{C}}{d s}\right|_{0} s_{I}^{\prime}
$$

where $s_{I}^{\prime}$ is the perturbed dimensionless interface position. Since $C$ at $s_{I}^{\prime}$ and $\bar{C}$ are both one, equation 7 becomes $\left.C^{\prime}\right|_{0}=-d \bar{C} /\left.d s\right|_{0} s_{I}^{\prime}=s_{I}^{\prime}$. Using a similar expansion for the concentration gradient, the perturbed interface position is shown to evolve according to

$$
\frac{d s_{I}^{\prime}}{d \tau}=-\frac{1}{3} f_{V} C_{0}\left(\left.\frac{\partial C^{\prime}}{\partial s}\right|_{0}+\left.\frac{d^{2} \bar{C}}{d s^{2}}\right|_{0} s_{I}^{\prime}\right)+A \nabla_{1}^{2} s_{I}^{\prime}
$$


where $A=a v_{d} / D_{V}$. The perturbed variables are represented as Fourier modes, e. g. $C^{\prime}=\hat{C}(z) \exp (\beta \tau+i \mathbf{k} \square \mathbf{x})$, where $\mathbf{k}$ is the dimensionless wavenumber vector with components $k_{x}$ and $k_{y}$. The solution for the amplitude of the perturbed concentration is found to be $\hat{C}=\hat{s}_{I} \exp \left[-\left(1+\sqrt{1+4 \beta+4 k^{2}}\right) s / 2\right]$. Substituting for the concentration gradient into the perturbed interface motion equation (4) leads to a quadratic algebraic equation relating the growth factor $\beta$ to system parameters,

$$
\frac{6 \beta}{f_{V} C_{0}}+\frac{6 A k^{2}}{f_{V} C_{0}}+1=\sqrt{1+4 \beta+4 k^{2}}
$$

The stability characteristics of the dissolving interface are determined by the roots of equation $5, \beta\left(A, f_{V} C_{0}, k\right)$. One of these roots is always negative, while the other becomes positive for certain parameter ranges, indicating unstable behavior. The domains of stability and instability are the regions respectively above and below the curve in the inset of Figure 7, showing the critical $A / f_{V} C_{0}$ as a function of dimensionless wavenumber. Instability is found at sufficiently small values of $A=a v_{d} / D_{V}$, which controls stabilizing flow at ridges. The critical value of $a$ therefore scales with the product of the vacancy diffusion length $D_{V} / v_{I}$, the vacancy contraction $f_{V}$, and the interface vacancy concentration $C_{0}$. As the measured tensile force indicates that $C_{0}$ is smaller than $10^{-2}$, the critical $a$ value is much smaller than the vacancy diffusion length. For all values of the system parameters, the inset of Figure 7 indicates unstable and stable behavior at low and high wavenumbers, respectively. This is because the vacancy 
diffusion length controls destabilizing large-scale vacancy diffusion, while the relatively smaller length parameter $a$ controls the stabilizing effect of enhanced dissolution at ridges.

Figure 7 shows examples of dispersion curves predicted by linear stability analysis. The growth factor $\beta$ is plotted against the wavenumber for three values of the parameter $A / f_{V} C_{0}$. Each curve exhibits a non-monotonic wavenumber dependence, with positive maxima at small $k$ and crossover to negative $\beta$ values at the critical wavenumbers in the inset plot. The fastest growing wavenumbers typically identify the wavelength of emergent patterns. Significantly, the pattern wavelength scales with the vacancy diffusion length, i. e. $\lambda \sim D_{V} / 2 \pi v_{I}$. This correspondence of length scales is the same as that implied by the experimentally observed relationship between wavelength and plastic layer thickness in Table 1. Patterns with the same $D_{V} / \nu \lambda$ ratio as that found experimentally are obtained near the limiting $A / f_{V} C_{0}$ ratio of 2 at large wavelength. In addition, the small values of $A$ associated with the pattern suggest that the magnitude of the effective capillary length $a$ is no larger than about $10 \mathrm{~nm}$. This estimate compares favorably with both the observed ridge radius of curvature and the capillary length estimated from the yield stress and surface free energy. The apparent agreement with experiment of both the pattern length scale and the capillary length is evidence that the dimpled pattern arises from plastic deformation accommodating tensile strain induced by vacancy diffusion.

The present morphological stability model predicts dimpled surface patterns in a given system when metal vacancies are generated at the surface. Since uniform corrosion processes produce similar dimpled or scalloped surface patterns on a variety of metals, 
the scope of vacancy-induced plastic flow may extend beyond aluminum [57-61]. If so, our approach of combined stress and topography measurements would be expected to reveal the expected scaling relationships between plastic layer thickness and pattern length scales. Vacancy-induced plastic deformation might also explain TEM observations of enhanced dislocation activity near dissolving surfaces on brass and stainless steel [62, 63]. These systems exhibit enhanced susceptibility to stress corrosion cracking, raising the possibility discussed above that vacancy injection may play a direct role in the SCC mechanism. Vacancies generated near crack surfaces during SCC would also efficiently trap absorbed hydrogen, dramatically enhancing the local $\mathrm{H}$ concentration so as to activate HEDE and HELP hydrogen embrittlement processes. In addition to mechanistic implications for SCC and HE, the present results show that corrosion processes can produce defective and presumably weakened surface layers that may be precursors for crack initiation. Detection of such layers, if possible, may provide valuable early indications of stress corrosion cracking susceptibility prior to the catastrophic propagation stage. 


\section{Conclusions}

This article reports stress measurements on Al sheet and foil samples during alkaline corrosion using phase-shifting curvature interferometry. The use of these bulk samples permitted study of material property effects on stress generation during extended corrosion exposures. The measurements reveal tensile force increases that scale with the yield strength. Coupled stress and topography changes strongly suggests that both are associated with plastic deformation. Spectral analysis of SEM images shows that the characteristic wavelength of the dimpled or scalloped surface topographic pattern produced by dissolution remains comparable to the plastic layer thickness interpreted from stress measurements. It was proposed that tensile stress in the metal is produced by lattice contraction associated by metal vacancies injected during dissolution. The driving force for vacancy injection arises from the low aluminum chemical potential and elevated hydrogen chemical potential, imposed respectively by the high electrochemical overpotential and hydrogen absorption rate during metal dissolution. The large overpotential is in turn associated with simultaneous $\mathrm{Al}$ and hydride oxidation reactions during corrosion. In support of the hypothesis that vacancies induce tensile stress and plastic deformation, we showed that vacancy diffusion controls the transient penetration of the plastic layer into the metal, and hence the time dependence of the measured force. Further, morphological stability analysis showed that the scaling relationship of the topography wavelength and plastic layer thickness is consistent with plastic deformation controlled by vacancy diffusion. 


\section{Acknowledgments}

This research was supported financially by the National Science Foundation (CMMI100748). Dr. G. R. Stafford (National Institute of Standards and Technology) performed electron backscatter diffraction measurements of the Al sheet sample. We also acknowledge valuable discussions with Professor Kai-Ming Ho (Iowa State University).

\section{References}

[1] J.R. Scully, G.A. Young, S.W. Smith. Hydrogen embrittlement of aluminum and aluminum-based alloys. in: Gangloff RP, Somerday BP, (Eds.). Gaseous hydrogen embrittlement of materials in energy technologies, vol. 1. Woodhead, Oxford, 2012. pp. 707-768.

[2] G. Lu, Q. Zhang, N. Kioussis, E. Kaxiras. Hydrogen-enhanced local plasticity in aluminum: An ab initio study, Phys. Rev. Lett. 87 (2001) art. no.-095501.

[3] S.W. Ciaraldi, J.L. Nelson, R.A. Yeske, E.N. Pugh. Studies of Hydrogen Embrittlement and Stress-Corrosion Cracking in Aluminum-Zinc-Magnesium Alloy. in: Bernstein IM, Thompson AW, (Eds.). Hydrogen Effects in Metals. The Metallurgical Society of AIME, Warrendale, PA, 1981. pp. 449-457.

[4] R.W. Revie, H.H. Uhlig. Effect of Applied Potential and Surface Dissolution on Creep Behavior of Copper, Acta Metall. 22 (1974) 619-627.

[5] K. Lian, E.I. Meletis. Environment-induced deformation localization during transgranular stress corrosion cracking, Corrosion 52 (1996) 347-355. 
[6] D.A. Jones. Localized surface plasticity during stress corrosion cracking, Corrosion 52 (1996) 356-362.

[7] M. Iwamoto, Y. Fukai. Superabundant vacancy formation in iron under high hydrogen pressures: Thermal desorption spectroscopy, Mater. T. JIM 40 (1999) 606-611. [8] G. Lu, E. Kaxiras. Hydrogen Embrittlement of Aluminum: The Crucial Role of Vacancies, Phys. Rev. Lett. 94 (2005) 155501/155501-155501/155504.

[9] K. Takai, H. Shoda, H. Suzuki, M. Nagumo. Lattice defects dominating hydrogen-related failure of metals, Acta Mater. 56 (2008) 5158-5167.

[10] K. Arioka, T. Miyamoto, T. Yamada, M. Aoki. Role of Cavity Formation in Crack Initiation of Cold-Worked Carbon Steel in High-Temperature Water, Corrosion 69 (2013) 487-496.

[11] H.W. Pickering, C. Wagner. Electrolytic Dissolution of Binary Alloys Containing a Noble Metal, J. Electrochem. Soc. 114 (1967) 698-706.

[12] L. Ismer, M.S. Park, A. Janotti, C.G. Van de Walle. Interactions between hydrogen impurities and vacancies in $\mathrm{Mg}$ and $\mathrm{Al}$ : A comparative analysis based on density functional theory, Phys. Rev. B 80 (2009) 184110.

[13] M. Ji, C.Z. Wang, K.M. Ho, S. Adhikari, K.R. Hebert. Statistical model of defects in Al-H system, Phys. Rev. B 81 (2010) 024105.

[14] H.K. Birnbaum, C. Buckley, F. Zeides, E. Sirois, P. Rozenak, S. Spooner, J.S. Lin. Hydrogen in aluminum, J. Alloys Compd. 253 (1997) 260-264.

[15] S. Parida, D. Kramer, C.A. Volkert, H. Roesner, J. Erlebacher, J. Weissmueller. Volume change during the formation of nanoporous gold by dealloying, Phys. Rev. Lett. 97 (2006). 
[16] S. Serebrinsky, E.A. Carter, M. Ortiz. A quantum-mechanically informed continuum model of hydrogen embrittlement, J Mech Phys Solids 52 (2004) 2403-2430. [17] Y. Sun, Q. Peng, G. Lu. Quantum mechanical modeling of hydrogen assisted cracking in aluminum, Phys Rev B 88 (2013).

[18] J. Song, W.A. Curtin. Atomic mechanism and prediction of hydrogen embrittlement in iron, Nat Mater 12 (2013) 145-151.

[19] O. Verners, G. Psofogiannakis, A.C.T. van Duin. Comparative molecular dynamics study of fcc-Al hydrogen embrittlement, Corros. Sci. 98 (2015) 40-49. [20] K.R. Hebert, J.H. Ai, G.R. Stafford, K.M. Ho, C.Z. Wang. Vacancy defects in aluminum formed during aqueous dissolution, Electrochim. Acta 56 (2011) 1806-1809. [21] J.W. Shin, G.R. Stafford, K.R. Hebert. Stress in aluminum induced by hydrogen absorption during cathodic polarization, Corros. Sci. 98 (2015) 366-371.

[22] H. Lu, K.W. Gao, W.Y. Chu. Determination of tensile stress induced by dezincification layer during corrosion for brass, Corros. Sci. 40 (1998) 1663-1670.

[23] T. Zhang, W.Y. Chu, K.W. Gao, L.J. Qiao. Study of correlation between hydrogen-induced stress and hydrogen embrittlement, Mater. Sci. Eng. A 347 (2003) 291-299.

[24] S. Adhikari, J. Ai, K. Hebert, K. Ho, C. Wang. Hydrogen in aluminum during alkaline corrosion, Electrochim. Acta 55 (2010) 5326-5331.

[25] K.R. Hebert, H.Q. Wu, T. Gessmann, K. Lynn. Positron annihilation spectroscopy study of interfacial defects formed by dissolution of aluminum in aqueous sodium hydroxide, J. Electrochem. Soc. 148 (2001) B92-B100. 
[26] R.C. Huang, K.R. Hebert, T. Gessmann, K.G. Lynn. Effect of impurities on interfacial void formation in aluminum, J. Electrochem. Soc. 151 (2004) B227-B232.

[27] S. Adhikari, K. Hebert, L. Chumbley, H. Chen, Y. Jean, A. Geiculescu. Interfacial Voids on Aluminum Created by Aqueous Dissolution, Electrochim. Acta 55 (2010) 6093-6100.

[28] J. Wang, P. Shrotriya, K.S. Kim. Surface residual stress measurement using curvature interferometry, Exp Mech 46 (2006) 39-46.

[29] O.O. Capraz, K.R. Hebert, P. Shrotriya. In Situ Stress Measurement During Aluminum Anodizing Using Phase-Shifting Curvature Interferometry, J. Electrochem. Soc. 160 (2013) D501-D506.

[30] J.R. Cahoon, W.H. Broughton, A.R. Kutzak. Determination of Yield Stress From Hardness Measurements, Metallurgical Transactions 2 (1971) 1979-1983.

[31] C.E. Caicedo-Martinez, E.V. Koroleva, G.E. Thompson, P. Skeldon, K. Shimizu, H. Habazaki, G. Hoellrigl. Surface nanotextures on aluminium, Surf. Interface Anal. 34 (2002) 405-408.

[32] E.V. Koroleva, G.E. Thompson, P. Skeldon, B. Noble. Crystallographic dissolution of high purity aluminium, Proceedings of the Royal Society a-Mathematical Physical and Engineering Sciences 463 (2007) 1729-1748.

[33] S. Prussin. Generation and distribution of dislocations by solute diffusion, J. Appl. Phys. 32 (1961) 1876-1881.

[34] H. Leth-Olsen, J.H. Nordlien, K. Nisancioglu. Formation of nanocrystalline surface layers by annealing and their role in filiform corrosion of aluminum sheet, $\mathrm{J}$. Electrochem. Soc. 144 (1997) L196-L197. 
[35] Z. Ashitaka, G.E. Thompson, P. Skeldon, G.C. Wood, K. Shimizu. The behavior of copper and lead during heat-treatment and surface treatment of aluminium capacitor, $\mathrm{J}$. Electrochem. Soc. 146 (1999) 1380-1385.

[36] C.E. Buckley, H.K. Birnbaum. Characterization of the charging techniques used to introduce hydrogen in aluminum, J. Alloys Compd. 330 (2002) 649-653.

[37] J.W. Shin, E. Chason. Compressive stress generation in Sn thin films and the role of grain boundary diffusion, Phys. Rev. Lett. 103 (2009) 056102.

[38] C.E. Buckley, H.K. Birnbaum, J.S. Lin, S. Spooner, D. Bellmann, P. Staron, T.J. Udovic, E. Hollar. Characterization of $\mathrm{H}$ defects in the aluminium-hydrogen system using small-angle scattering techniques, J. Appl. Crystallogr. 34 (2001) 119-129.

[39] A. Seeger. Investigation of point defects in equilibrium concentrations with particular reference to positron annihilation techniques, Journal of Physics F: Metal Phys. 3 (1973) 248-295.

[40] H. Gunaydin, S.V. Barabash, K.N. Houk, V. Ozolins. First-principles theory of hydrogen diffusion in aluminum, Phys. Rev. Lett. 101 (2008) 075901.

[41] D. Zang, P. Maroevic, R.B. McLellan. Hydrogen-induced vacancies on metal surfaces, J. Phys. Chem. Solids 60 (1999) 1649-1654.

[42] Y. Fukai. Formation of superabundant vacancies in M-H alloys and some of its consequences. A review, J. Alloys Compd. 356-357 (2003) 263-269.

[43] K.R. Hebert. Trapping of Hydrogen Absorbed in Aluminum during Corrosion, Electrochim. Acta 168 (2015) 199-205. 
[44] A. Metsue, A. Oudriss, X. Feaugas. Hydrogen solubility and vacancy concentration in nickel single crystals at thermal equilibrium: New insights from statistical mechanics and ab initio calculations, J. Alloys Compd. 656 (2016) 555-567. [45] K.E. Heusler, W. Allgaier. Kinetics of the dissolution of aluminum in alkaline solutions, Werkst. Korros. 22 (1971) 297-302.

[46] S. Adhikari, J.J. Lee, K.R. Hebert. Formation of aluminum hydride during alkaline dissolution of aluminum, J. Electrochem. Soc. 155 (2008) C16-C21.

[47] Y. Zhang, J.H. Ai, A.C. Hillier, K.R. Hebert. Atom Probe Tomography Characterization of Thin Copper Layers on Aluminum Deposited by Galvanic Displacement, Langmuir 28 (2012) 1673-1677.

[48] G.G. Perrault. Role of Hydrides in the Equilibrium of Aluminum in AqueousSolutions, J. Electrochem. Soc. 126 (1979) 199-204.

[49] S. Adhikari, K.R. Hebert. Factors controlling the time evolution of the corrosion potential of aluminum in alkaline solutions, Corros. Sci. 50 (2008) 1414-1421.

[50] Y. Zhang, J. Ai, A.C. Hillier, K.R. Hebert. Atom Probe Tomography

Characterization of Thin Copper Layers on Aluminum Deposited by Galvanic Displacement, Langmuir 28 (2012) 1673-1677.

[51] M. Pourbaix. Atlas of Electrochemical Equilibria in Aqueous Solutions, NACE International, Houston, 1974.

[52] F.P. Ford, G.T. Burstein, T.P. Hoar. Bare Surface-Reaction Rates and Their Relation to Environment Controlled Cracking of Aluminum-Alloys .1. Bare SurfaceReaction Rates on Aluminum 7-Weight-Percent Magnesium in Aqueous-Solutions, J. Electrochem. Soc. 127 (1980) 1325-1331. 
[53] N.L. Peterson, S.J. Rothman. Impurity Diffusion in Aluminum, Phys Rev B 1 (1970) 3264-3273.

[54] R.W. Siegel. Atomic Defects and Diffusion in Metals. in: Takamura JI, Doyama M, Kiritani M, (Eds.). Point Defects and Defect Interactions in Metals. North-Holland, Amsterdam, 1982. pp. 533-540.

[55] S.M. Myers, P. Nordlander, F. Besenbacher, J.K. Norskov. Theoretical Examination of the Trapping of Ion-Implanted Hydrogen in Metals, Phys. Rev. B 33 (1986) 854-863.

[56] K.R. Hebert, G. Zhang, K.-M. Ho, C.-Z. Wang. Modeling electrochemical and metal-phase processes during alkaline aluminum corrosion, Electrochim. Acta 58 (2011) 203-208.

[57] S. Tanaka, T. Iwatani, N. Hirose, T. Tanaki. Effect of hydrogen on the formation of porous TiO2 in alkaline solution, J. Electrochem. Soc. 149 (2002) F186-F190.

[58] S.-i. Tanaka, H. Tobimatsu, Y. Maruyama, T. Tanaki, G. Jerkiewicz. Preparation and Characterization of Microporous Layers on Titanium, Acs Applied Materials \& Interfaces 1 (2009) 2312-2319.

[59] C. Kruse, M.W. Finnis, J.S. Lin, M.C. Payne, V.Y. Milman, A. DeVita, M.J. Gillan. First-principles study of the atomistic and electronic structure of the niobiumalpha-alumina(0001) interface, Philos. Mag. Lett. 73 (1996) 377-383.

[60] S. Singh, W.R.T. Barden, P. Kruse. Nanopatterning of Transition Metal Surfaces via Electrochemical Dimple Array Formation, Acs Nano 2 (2008) 2453-2464. 
[61] A.D. Pauric, S.A. Baig, A.N. Pantaleo, Y. Wang, P. Kruse. Sponge-Like Porous Metal Surfaces from Anodization in Very Concentrated Acids, J. Electrochem. Soc. 160 (2013) C12-C18.

[62] B.A. Gu, J.W. Zhang, F.R. Wang, W.Y. Chu. The in-Situ Tem Observation of Corrosion Facilitating Dislocation Emission, Multiplication and Motion for Brass, Scripta Metall Mater 32 (1995) 637-640.

[63] J.X. Li, W.Y. Chu, Y.B. Wang, L.J. Qiao. In situ TEM study of stress corrosion cracking of austenitic stainless steel, Corros. Sci. 45 (2003) 1355-1365. 
Table 1. Estimates of vacancy diffusivity from stress measurements for Al sheet.

Symbols: $C \mathrm{NaOH}$ concentration; $v_{d}$ dissolution velocity; ts time to force plateau; $F_{W S}$ force at plateau; $D_{V}(t s)$ vacancy diffusivity estimated from $t s ; D_{V}\left(F_{w s}\right)$ vacancy diffusivity estimated from $F_{W S}$.

\begin{tabular}{|l|l|l|l|l|l|l|}
\hline$C$ & $v_{d}$ & $t_{s}$ & $F w s$ & $D_{V}(t s)$ & $D_{V}\left(F_{W S}\right)$ & $D_{V} / V_{d} \lambda$ \\
$M$ & $\mathrm{~nm} / \mathrm{min}$ & $\min$ & $\mathrm{N} / \mathrm{m}$ & $\mathrm{m}^{2} / \mathrm{s}$ & $\mathrm{m}^{2} / \mathrm{s}$ & \\
\hline 0.1 & 31 & 7.0 & 31 & $2.8 \times 10^{-17}$ & $4.2 \times 10^{-17}$ & 0.13 \\
\hline 0.5 & 71 & 10 & 64 & $2.1 \times 10^{-16}$ & $3.2 \times 10^{-16}$ & 0.35 \\
\hline 1.0 & 131 & 16 & 90 & $1.1 \times 10^{-15}$ & $1.7 \times 10^{-15}$ & 0.60 \\
\hline
\end{tabular}




\section{List of Figures}

Figure 1. Effect of $\mathrm{NaOH}$ concentration on force per width evolution during open-circuit alkaline etching.

Figure 2. Force evolution of $\mathrm{Al}$ foil $(\mathbf{O})$, sheet $(\mathbf{\Delta})$ and rolled sheet $(\mathrm{O})$ during dissolution in $1 \mathrm{M} \mathrm{NaOH}$. The main panel shows examples of force transients of samples with different yield stress. The inset demonstrates linear relationship between plateau force $F_{W S}$ and yield stress $\sigma_{Y}$ (circular symbols are used for clarity). The slope of the linear regression fit is $1.00 \mu \mathrm{m}$.

Figure 3. Image analysis of dissolution topography. (a), (b), SEM images after dissolution in $1 \mathrm{M} \mathrm{NaOH}$ for 5 and 20 min, respectively. (c) Image representing power spectral density of SEM image in (b). (d) Power spectral density plotted along the line in (c). Arrow indicates the characteristic pattern wavelength.

Figure 4. Characteristic wavelengths of dimple patterns after alkaline dissolution of $\mathrm{Al}$ in $1 \mathrm{M} \mathrm{NaOH}(\bullet), 0.5 \mathrm{M}(\boldsymbol{\square}, \square)$ and $0.1 \mathrm{M} \mathrm{NaOH}(\diamond, \diamond)$. The main panel shows wavelengths (left axis) of samples after different dissolution times. Solid symbols are plotted at the measurement time, open symbols at the time of the beginning of the force plateau. Force transient in $1 \mathrm{M} \mathrm{NaOH}$ is shown on right axis. The inset shows the relationship of force per width and wavelength, with the solid line a linear regression fit. 
Figure 5. Evolution of normalized force of various samples during dissolution in $1 \mathrm{M}$ $\mathrm{NaOH}$. Normalized force is the measured force per width divided by the yield stress.

Figure 6. Vacancy concentration profiles calculated by the one-dimensional diffusion model. The ordinate and abscissa are respectively is the vacancy concentration normalized with the surface concentration $C_{0}$, and the dimensionless depth relative to the dissolving interface. The parameter is dimensionless time (see text for definitions).

Figure 7. Results of linear stability analysis. Main panel shows examples of dispersion curves for various values of $A / f_{V} C_{0}$ (parameter in plot). Values of $f_{V}$ and $C_{0}$ are 0.35 and 0.001. Inset illustrates critical conditions for instability. Unstable and stable behavior respectively are predicted below and above the curve. 


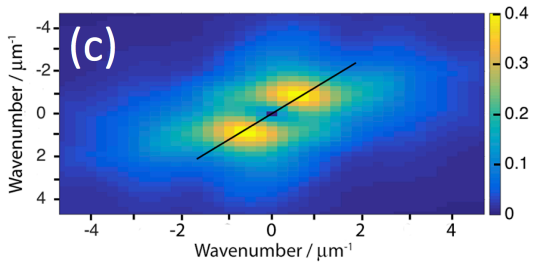

(b)

\section{$3 \mu \mathrm{m}$}

HV WD det mag g opot prossure
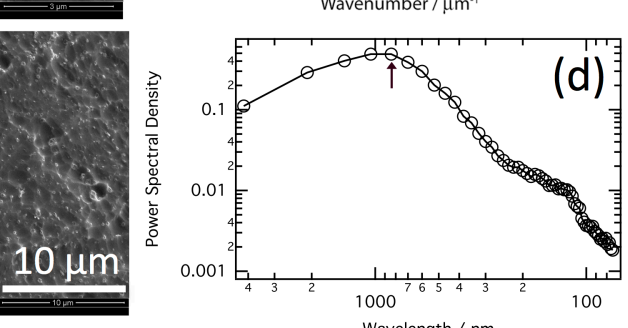
Figure 5

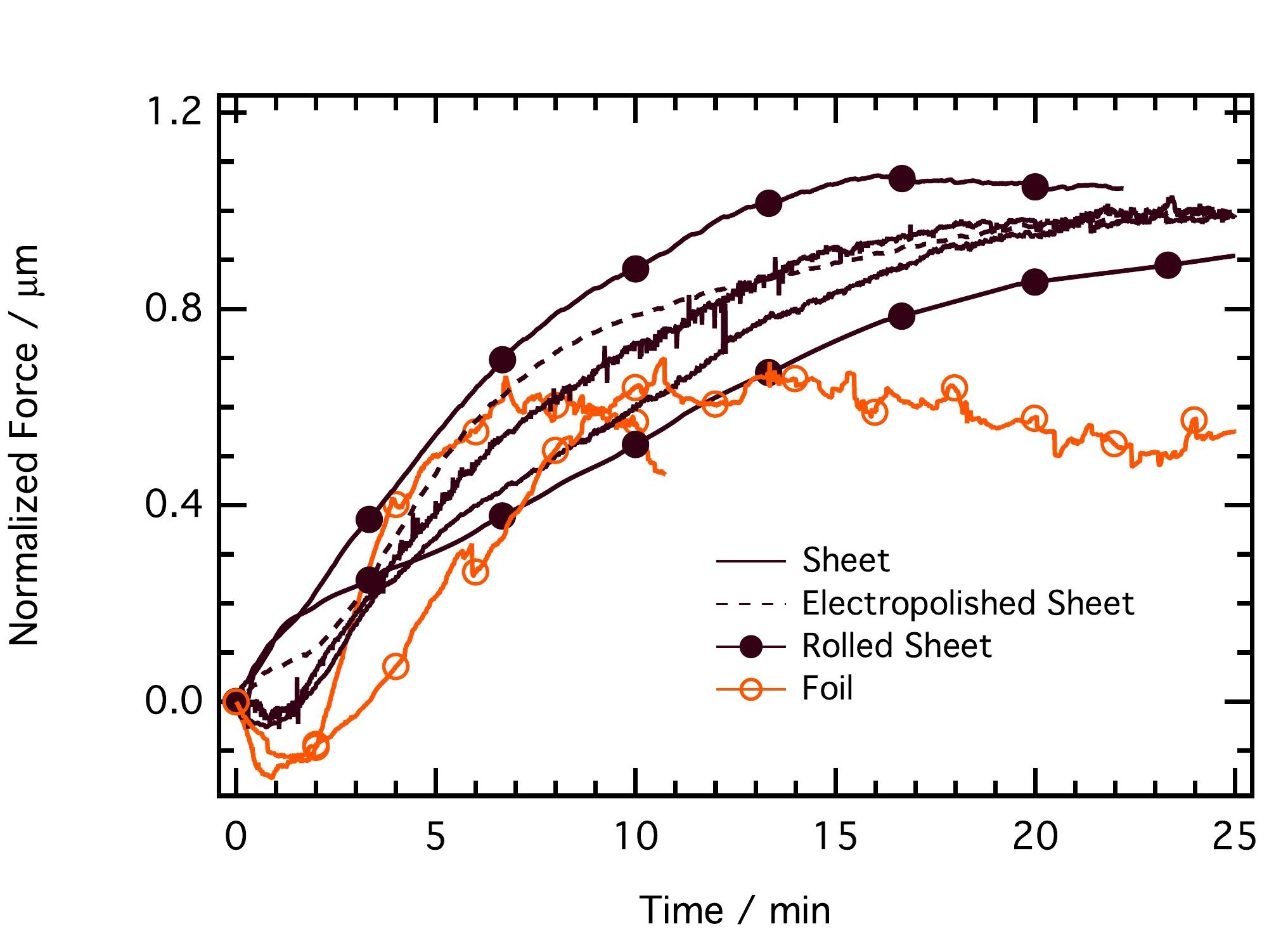

Figure

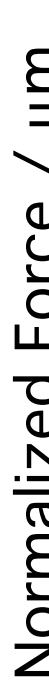

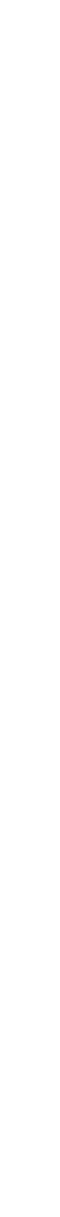

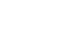



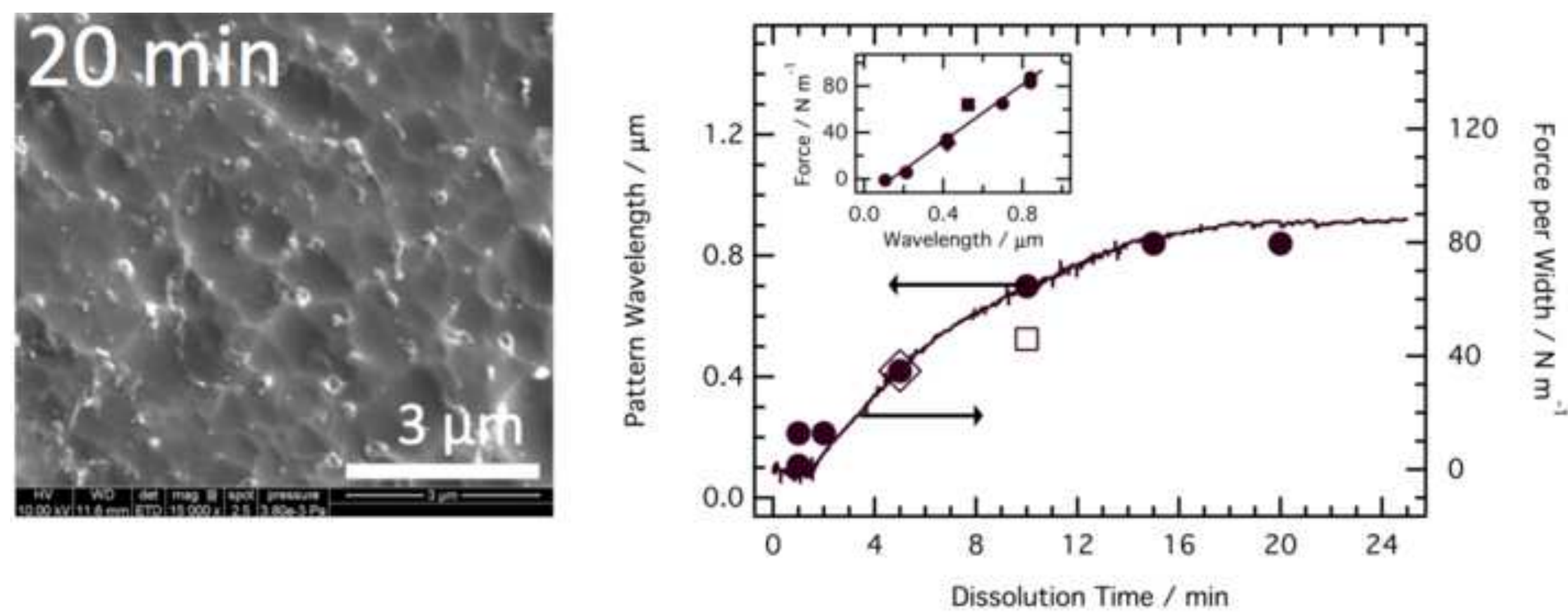